\title{
An implementation of the Owen value*
}

\author{
Gustavo Bergantiños and Juan J. Vidal-Puga ${ }^{\dagger}$
}

\begin{abstract}
We present an implementation of the Owen value (Owen, 1977), inspired by the bidding mechanism introduced by Pérez-Castrillo and Wettstein (2001). The idea is the following: first players of each coalition play the bidding mechanism trying to obtain the resources of the coalition. Later, players who obtained the resources play the bidding mechanism in order to share the surplus.
\end{abstract}

\section{Introduction}

The Shapley value (Shapley, 1953) is one of the most important solution concepts in cooperative game theory. Since the paper of Shapley was published, many authors have been studying this value. For instance, Myerson (1980) characterizes it using the property of balanced contributions. Hart and Mas-Colell (1989) do so by using the potential and the property of consistency. Moreover, the Shapley value has been used successfully in cost allocation problems and the analysis of political situations.

Another important aspect of a normative solution is the non-cooperative foundation, or implementation. The idea is to prove that agents can reach the cooperative solution through a non-cooperative behavior. Indeed, given the cooperative game, a noncooperative game is associated in such a way that the outcome of some kind of equilibrium of the non-cooperative game coincides with the solution of the cooperative game. There are several implementations of the Shapley value (for instance, Gul (1989), Hart and Mas-Colell (1996), and Pérez-Castrillo and Wettstein (2001)).

Owen (1977) studied situations in which players are partitioned into groups. In this context Owen introduced the Owen value, which is a generalization of the Shapley value.

*DOI: www.doi.org/10.1016/S0899-8256(03)00043-5. Creative Commons Attribution NonCommercial No Derivatives License.

${ }^{\dagger}$ Financial support from the Spanish government and the Xunta de Galicia through grants PB980613-C02-01 and PGIDT00PXI30001PN is gratefully acknowledged. 
Later, several authors extended other results of the Shapley value to the Owen value. Calvo, Lasaga, and Winter (1996) extended the results about balanced contributions, and Winter (1992) studied the results about the potential and consistency. Also, the Owen value has been used in cost allocation problems (Vázquez-Brage, van den Nouweland, and Garcia-Jurado (1997)) and political situations (Carreras and Owen (1988)).

Nevertheless, the non-cooperative foundation of the Shapley value has not been extended to the Owen value. In this paper we extend the results obtained by Pérez-Castrillo and Wettstein (2001) and we implement the Owen value.

Given a cooperative game, Pérez-Castrillo and Wettstein (2001) define a non-cooperative game called the "bidding mechanism". They prove that the payoff of all subgame perfect Nash equilibria outcomes coincides with the Shapley value of the cooperative game. In the bidding mechanism there are three stages. In Stage 1, players bid to become the proposer, where bids can be negative or positive. The player with the highest "net bid" (the difference between the sum of the bids he makes to the others minus the sum of the bids the others make to him) becomes the proposer and pays the bid to the other players. In Stage 2, the proposer makes an offer to the other players. In Stage 3, the other players answer the offer. If everybody accepts the offer, the grand coalition is formed, the proposer pays to the other players according to the offer, and obtains all the resources of the grand coalition. If some player rejects the offer, then the proposer is removed obtaining his own resources. The rest of the players play the bidding mechanism among themselves.

Given a cooperative game, we define a non-cooperative game called the "coalitional bidding mechanism" and we prove that the payoff of all subgame perfect Nash equilibria outcomes coincides with the Owen value of the cooperative game.

The coalitional bidding mechanism has two rounds. Assume that the coalition structure is $\left\{C_{1}, \ldots, C_{m}\right\}$. In Round 1 , the members of any coalition $C_{l}$, independently of the other coalitions, play the bidding mechanism among themselves. Then for any coalition $C_{l}$ we can find a player, called the representative, who obtains the resources of coalition $C_{l}$, or a subcoalition of $C_{l}$ if some player is removed because his offer was rejected. In Round 2, the representatives play the bidding mechanism among themselves, taking into account the resources obtained in Round 1.

The paper is organized as follows. In Section 2 we present the notation and definitions. In Section 3 we define formally the coalitional bidding mechanism and we prove that it implements the Owen value. Finally, in Section 4 we present some concluding remarks. 


\section{The model}

First, we introduce the notation used throughout this paper. Let $(N, v)$ be a game with transferable utility (TU game), where $N=\{1,2, \ldots, n\}$ is the set of players and $v$ is the characteristic function, which assigns a real number $v(S)$ to every coalition $S \subset N$. We assume that $v(\varnothing)=0$. Following usual practice, we often refer to "the game $v$ " instead of "the game $(N, v)$ ". We denote by $T U(N)$ the set of all TU games on the set of players $N$. We denote by $T U$ the set of all TU games

For each game $v$ and $S \subset N$ we denote by $v_{\mid S}$ the game $v$ restricted to $S\left(v_{S}(T)=v(T)\right.$ for any $T \subset S)$. Moreover, $v_{-i}=v_{\mid N \backslash\{i\}}$.

A coalition structure for $N$ is a partition $C=\left\{C_{1}, \ldots, C_{m}\right\}, i$. e. $C_{k} \cap C_{l}=\varnothing$ if $k \neq l$ and $\bigcup_{l=1}^{m} C_{l}=N$. We assume that a coalition structure $C=\left\{C_{1}, \ldots, C_{m}\right\}$ is given and fixed. Given $S \subset N$ we denote by $C_{\mid S}$ the restriction of $C$ to the members of coalition $S$, i. e. $C_{S}=\left\{C_{l} \cap S \mid C_{l} \in C\right.$ and $\left.C_{l} \cap S \neq \varnothing\right\}$. Moreover, $C_{-i}=C_{\mid N \backslash\{i\}}$. We denote by $T U(N, C)$ the set of all triples $(N, C, v)$ where $N$ is the set of players, $C$ is a coalition structure for $N$, and $v$ is a characteristic function. We denote by $C T U$ the set of all triples $(N, C, v)$.

To each coalition structure $C$ and each game $v$ we define by $v^{C}$ the game induced by $v$ by considering the coalitions of $C$ as players. Then for any $Q \subset C, v^{C}(Q)=v\left(\cup_{C_{l} \in Q} C_{l}\right)$.

Let $\Omega(N)$ be the set of all permutations on $N$. We say that $\pi \in \Omega(N)$ is admissible with respect to the coalition structure $C$ if for any $i, j, k \in N, i, k \in C_{l} \in C$, and $\pi(i)<$ $\pi(j)<\pi(k)$ imply that $j \in C_{l}$, where $\pi(i), \pi(j), \pi(k)$ denote the position of $i, j$, and $k$ in the permutation $\pi$. We denote by $\Omega(N, C)$ the set of all admissible permutations on $N$ with respect to $C$.

We say that $v$ is: superadditive if $v(S)+v(T) \leq v(S \cup T)$ when $S \cap T=\varnothing$, strictly superadditive if $v(S)+v(T)<v(S \cup T)$ when $S \cap T=\varnothing$, zero-monotonic if $v(S)+v(\{i\}) \leq$ $v(S \cup\{i\})$ when $i \notin S$, and strictly zero-monotonic if $v(S)+v(\{i\})<v(S \cup\{i\})$ when $i \notin S$. Note that if the game is superadditive (strictly) then it is zero-monotonic (strictly).

Given $(N, C, v) \in T U(N, C)$ the Owen value (Owen, 1977) is defined as :

$$
\phi_{i}(N, C, v)=\frac{1}{|\Omega(N, C)|} \sum_{\pi \in \Omega(N, C)}\left[v\left(P_{i}^{\pi} \cup\{i\}\right)-v\left(P_{i}^{\pi}\right)\right] \quad \text { for all } i \in N
$$

where $P_{i}^{\pi}=\{j \in N \mid \pi(j)<\pi(i)\}$ and $|\Omega(N, C)|$ denotes the cardinality of the set $\Omega(N, C)$.

If $C=\{\{1\}, \ldots,\{n\}\}$ or $C=\{N\}$ then the Owen value is given by

$$
\phi_{i}(N, C, v)=\sum_{S \subseteq N \backslash\{i\}} \frac{|S| !(n-|S|-1) !}{n !}[v(S \cup\{i\})-v(S)] \text { for all } i \in N
$$


which coincides with the Shapley value of the game $v$. Usually we denote by $\phi(N, v)$ the Shapley value of $v$.

It is well known that for any triple $(N, C, v)$ and for any $C_{l} \in C, \sum_{i \in C_{l}} \phi_{i}(N, C, v)=$ $\phi_{C_{l}}\left(C, v^{C}\right)$ where $\phi_{C_{l}}\left(C, v^{C}\right)$ denotes the Shapley value of $C_{l}$ in the game $v^{C}$.

A value on $G \subset C T U$ is a map $\varphi: G \longrightarrow \mathbb{R}^{N}$.

We say that a value $\varphi$ on $G$ satisfies:

Efficiency if for any $(N, C, v) \in G, \sum_{i \in N} \varphi_{i}(N, C, v)=v(N)$.

Additivity if for any $\left(N, C, v_{1}\right),\left(N, C, v_{2}\right) \in G$ and $i \in N$,

$$
\varphi_{i}\left(N, C, v_{1}+v_{2}\right)=\varphi_{i}\left(N, C, v_{1}\right)+\varphi_{i}\left(N, C, v_{2}\right)
$$

where $\left(v_{1}+v_{2}\right)(S)=v_{1}(S)+v_{2}(S)$ for any $S \subset N$.

Balanced contributions among players if for any $(N, C, v) \in G$ and $i, j \in C_{l} \in C$,

$$
\varphi_{i}(N, C, v)-\varphi_{i}\left(N \backslash\{j\}, C_{-j}, v_{-j}\right)=\varphi_{j}(N, C, v)-\varphi_{j}\left(N \backslash\{i\}, C_{-i}, v_{-i}\right) .
$$

Balanced contributions among coalitions if for any $(N, C, v) \in G$ and $C_{k}, C_{l} \in C$,

$$
\begin{aligned}
& \sum_{i \in C_{k}} \varphi_{i}(N, C, v)-\sum_{i \in C_{k}} \varphi_{i}\left(N \backslash C_{l}, C_{\mid N \backslash C_{l}}, v_{\mid N \backslash C_{l}}\right)= \\
& \sum_{i \in C_{l}} \varphi_{i}(N, C, v)-\sum_{i \in C_{l}} \varphi_{i}\left(N \backslash C_{k}, C_{\mid N \backslash C_{k}}, v_{\mid N \backslash C_{k}}\right) .
\end{aligned}
$$

Owen (1977) proved that the Owen value satisfies efficiency and additivity. Later, Calvo et al. (1996) proved that also satisfies balanced contributions among players and coalitions.

\section{The coalitional bidding mechanism}

Given a cooperative game $(N, v)$, Pérez-Castrillo and Wettstein (2001) design a noncooperative game, called the bidding mechanism. They prove that the payoff of any subgame perfect Nash equilibrium (SPNE from now) of this mechanism always coincide with the Shapley value of the cooperative game $(N, v)$. Thus, this mechanism implements the Shapley value in subgame perfect Nash equilibria.

In this section we extend the bidding mechanism to cooperative games with a coalition structure. The idea is quite simple. There are Round 1 and Round 2, and Round 1 contains stages 1 through 3 . In Round 1, players in the same coalition play the bidding mechanism in order to obtain the resources of this coalition (or a subcoalition if some 
player is removed). The player who obtains the resources is called the "representative". In Round 2, the representatives play the bidding mechanism with the resources obtained in Round 1.

We now present the coalitional bidding mechanism (CBM) formally.

If there is only one player $i$, he obtains $v(\{i\})$. Assume now that we know the rules of the coalitional bidding mechanism when played by at most $n-1$ players. Then, for a set of players $N=\{1, \ldots, n\}$ and coalition structure $C=\left\{C_{1}, \ldots, C_{m}\right\}$, the CBM proceeds as follows.

1. Round 1. In this round, the players of any coalition $C_{l} \in C$ play the bidding mechanism trying to obtain the resources of $C_{l}$. Formally, if there is only one player $i$, then this player has his resources. Assume now that we know the rules when played by at most $\left|C_{l}\right|-1$ players. For $C_{l}$ proceeds as follows.

(a) Stage 1. Each player $i \in C_{l}$ makes bids $b_{j}^{i} \in \mathbb{R}$ for every $j \in C_{l} \backslash\{i\}$. For each $i \in C_{l}$, we take $B^{i}=\sum_{j \in C_{l} \backslash\{i\}} b_{j}^{i}-\sum_{j \in C_{l} \backslash\{i\}} b_{i}^{j}$. Assume that $\alpha_{l}=\operatorname{argmax}_{i}\left\{B^{i}\right\}$. In the case of a non-unique maximizer, $\alpha_{l}$ is randomly chosen among the maximizing indices.

(b) Stage 2. Player $\alpha_{l}$, called the proposer, makes an offer $y_{i}^{\alpha_{l}}$ to every player $i \in C_{l} \backslash\left\{\alpha_{l}\right\}$.

(c) Stage 3. The players of $C_{l} \backslash\left\{\alpha_{l}\right\}$, sequentially, either accept or reject the offer. If a rejection is encountered, we say the offer is rejected. Otherwise, we say the offer is accepted.

The coalitions of $C$ play sequentially in the order $C_{1}, \ldots, C_{m}$ until we find $C_{l_{0}}$ and $\alpha_{l_{0}}$ such that the offer of $\alpha_{l_{0}}$ is rejected or for any $C_{l} \in C$ the offer of $\alpha_{l}$ is accepted. In the first case, player $\alpha_{l_{0}}$ pays $b_{i}^{\alpha_{l}}$ to every player $i \in C_{l} \backslash\left\{\alpha_{l}\right\}$ and leaves the non-cooperative game obtaining $v\left(\left\{\alpha_{l_{0}}\right\}\right)-\sum_{i \in C_{l_{0}} \backslash\left\{\alpha_{l_{0}}\right\}} b_{i}^{\alpha_{l_{0}}}$. All players other than $\alpha_{l_{0}}$ proceed to play the CBM with $\left(N^{\prime}, C^{\prime}, v^{\prime}\right)$ where $N^{\prime}=N \backslash\left\{\alpha_{l_{0}}\right\}, C^{\prime}=C_{-\alpha_{l_{0}}}$, and $v^{\prime}=$ $v_{-\alpha_{l_{0}}}$. Any player $i \in C_{l_{0}} \backslash\left\{\alpha_{l_{0}}\right\}$ obtains as final payoff the sum of the bid received, $b_{i}^{\alpha_{l_{0}}}$, and the payoff outcome of the mechanism corresponding to $\left(N^{\prime}, C^{\prime}, v^{\prime}\right)$. Any player $i \in N^{\prime} \backslash C_{l_{0}}$ obtains as final payoff the payoff outcome of the mechanism corresponding to $\left(N^{\prime}, C^{\prime}, v^{\prime}\right)$.

In the second case, for any $C_{l} \in C$, player $\alpha_{l}$ pays $b_{i}^{\alpha_{l}}+y_{i}^{\alpha_{l}}$ to every player $i \in C_{l} \backslash\left\{\alpha_{l}\right\}$ and becomes the "representative" of coalition $C_{l}$. This means that player $\alpha_{l}$ goes to 
Round 2 with all the resources of $C_{l}$. Moreover, the payoff obtained by this player in this round is $p_{\alpha_{l}}^{1}=-\sum_{i \in C_{l} \backslash\left\{\alpha_{l}\right\}}\left(b_{i}^{\alpha_{l}}+y_{i}^{\alpha_{l}}\right)$. Any other player $i \in C_{l} \backslash\left\{\alpha_{l}\right\}$ leaves the non-cooperative game obtaining a final payoff of $b_{i}^{\alpha_{l}}+y_{i}^{\alpha_{l}}$.

After finishing Round 1, for any $C_{l} \in C$ we can find the representative (denoted by $r_{l}$ ) of this coalition. When there is only one player in a coalition, he becomes the representative of himself. Moreover, we denote by $C_{l}^{r}$ the set of players whose resources are obtained by player $r_{l}$. Notice that $C_{l} \backslash C_{l}^{r}$ is the set of removed proposers in $C_{l}$. Of course $C_{l}^{r} \subset C_{l}$ and $r_{l} \in C_{l}^{r}$.

2. Round 2. The representatives play the bidding mechanism (Pérez-Castrillo and Wettstein, 2001) associated with the game $\left(N^{r}, v^{r}\right)$ where $N^{r}=\left\{r_{1}, \ldots, r_{m}\right\}$ and for any $S \subset N^{r}, v^{r}(S)=v\left(\bigcup_{r_{l} \in S} C_{l}^{r}\right)$. For any representative $r_{l}$, we denote by $p_{r_{l}}^{2}$ the payoff obtained by $r_{l}$ in Round 2 .

The final payoff obtained by any representative $r_{l}$ is the sum of the payoffs obtained in both rounds, i. e. $p_{r_{l}}^{1}+p_{r_{l}}^{2}$.

We must note that the CBM terminates in a finite number of moves.

Gul (1989) analyzed a cooperative game where random meetings between two agents occur. At each meeting, a player (randomly chosen) makes an offer to the other. If this offer is accepted, the proposer buys the resources of the other player. In the bidding mechanism played by any coalition the situation is, in some way, similar. There is also a player $\left(\alpha_{l}\right)$ who makes an offer trying to obtain the resources of the rest of players $\left(C_{l} \backslash\left\{\alpha_{l}\right\}\right)$. The differences are that in the bidding mechanism it could be possible that more than two players are involved and, moreover, the proposer is not randomly chosen.

In Round 1, we assume that coalitions play the bidding mechanism independently. Moreover, when Stage 3 of the bidding mechanism of some coalition $C_{l}$ ends, players of $C_{l}$ must announce to the other coalitions if they have an agreement or not. If they have not an agreement (in this case the proposer, $\alpha_{l}$, is removed), then we suppose that the coalitions that have already achieved an agreement can renegotiate it. Assume that coalition $C_{p}$ achieves an agreement but after knowing that player $\alpha_{l}$ has been removed some player in $C_{p}$ thinks that the agreement is not good. Then the agreement achieved is cancelled. In such case, the players of coalition $C_{p}$ play the bidding mechanism again.

In Round 2, the representatives of every coalition play the bidding mechanism with the resources obtained in Round 1. This round coincides with the bidding mechanism of Pérez-Castrillo and Wettstein (2001). 
Remark 1. In Round 1, we assume that coalitions play the bidding mechanism in the order $C_{1}, \ldots, C_{m}$. Our results are independent of the order in which coalitions play the bidding mechanism. Moreover, if the order is chosen according to some probability distribution over the set of all possible orders, our results are still valid and independent of the probability distribution.

Remark 2. Assume that the offer of player $\alpha_{l}$ is accepted for any $l<l_{0}$, but the offer of $\alpha_{l_{0}}$ is rejected. Then a new subgame begins, which coincides with the CBM associated to $\left(N \backslash\left\{\alpha_{l_{0}}\right\}, C_{-\alpha_{l_{0}}}, v_{-\alpha_{l_{0}}}\right)$.

Remark 3. According to the CBM, the agreement achieved by every coalition $C_{l}$ with $l<l_{0}$ is, somehow, cancelled. We can include in the CBM an intermediate step between the rejection of the offer of $\alpha_{l_{0}}$ and the CBM applied to $\left(N \backslash\left\{\alpha_{l_{0}}\right\}, C_{-\alpha_{l_{0}}}, v_{-\alpha_{l_{0}}}\right)$. Assume that for any coalition $C_{l}, l \neq l_{0}$, the players of $C_{l}$ vote whether they prefer to continue with the agreement achieved or not. If at least one player wants to cancel the agreement, it is cancelled. It is easy to check that all the results obtained in this paper are also valid if we include this intermediate step.

Before the characterization of the SPNE outcomes of the coalitional bidding mechanism we need the following result.

Proposition 1. Given a triple $(N, C, v)$ such that $v$ is zero-monotonic, $j \in C_{l} \in C$, and $\{j\} \neq C_{l}$ then

$$
\sum_{i \in C_{l}} \phi_{i}(N, C, v) \geq \sum_{i \in C_{l} \backslash\{j\}} \phi_{i}\left(N \backslash\{j\}, C_{-j}, v_{-j}\right)+v(\{j\}) .
$$

Proof. We take $M=\{1, \ldots, m\}$ and define the following games on $M: w(T)=v\left(\cup_{a \in T} C_{a}\right)$ for any $T \subset M ; w_{1}(T)=v\left(\bigcup_{a \in T} C_{a}\right)$ if $l \notin T$ and $w_{1}(T)=v\left(\bigcup_{a \in T \backslash\{l\}} C_{a} \cup\left(C_{l} \backslash\{j\}\right)\right)$ if $l \in T ; w_{2}(T)=0$ if $l \notin T$ and $w_{2}(T)=v(\{j\})$ if $l \in T$; and $w^{\prime}=w_{1}+w_{2}$.

Since $v$ is zero-monotonic we obtain that $w(T)=w^{\prime}(T)$ if $l \notin T$ and $w(T) \geq w^{\prime}(T)$ if $l \in T$. Hence $\phi_{l}(M, w) \geq \phi_{l}\left(M, w^{\prime}\right)$. Since the Shapley value satisfies additivity we have $\phi_{l}\left(M, w^{\prime}\right)=\phi_{l}\left(M, w_{1}\right)+\phi_{l}\left(M, w_{2}\right)$.

We know that $\phi_{l}(M, w)=\sum_{i \in C_{l}} \phi_{i}(N, C, v), \phi_{l}\left(M, w_{1}\right)=\sum_{i \in C_{l} \backslash\{j\}} \phi_{i}\left(N \backslash\{j\}, C_{-j}, v_{-j}\right)$, and $\phi_{l}\left(M, w_{2}\right)=v(\{j\})$. This concludes the proof.

Remark 4. Using similar arguments to those used in the proof of Proposition 1 we 
can prove that if $v$ is strictly zero-monotonic, $j \in C_{l} \in C$ and $\{j\} \neq C_{l}$, then

$$
\sum_{i \in C_{l}} \phi_{i}(N, C, v)>\sum_{i \in C_{l} \backslash\{j\}} \phi_{i}\left(N \backslash\{j\}, C_{-j}, v_{-j}\right)+v(\{j\}) .
$$

First, we prove that the Owen value is the payoff of an SPNE outcome.

Proposition 2. Given a triple $(N, C, v)$ where $v$ is superadditive, the Owen value $\phi(N, C, v)$ is the payoff of an SPNE outcome of the coalitional bidding mechanism.

Proof. If there is only a player the result is trivial. Assume that the result holds with at most $n-1$ players.

We consider the following strategies.

- Round 1. First, we define the strategies in the bidding mechanism associated to any $C_{l} \in C$.

Stage 1. For any $i \in C_{l}, b_{j}^{i}=\phi_{j}(N, C, v)-\phi_{j}\left(N \backslash\{i\}, C_{-i}, v_{-i}\right)$ for any $j \in C_{l} \backslash\{i\}$.

Stage 2. Player $\alpha_{l}$, the proposer, offers $y_{j}^{\alpha_{l}}=\phi_{j}\left(N \backslash\left\{\alpha_{l}\right\}, C_{-\alpha_{l}}, v_{-\alpha_{l}}\right)$ to every $j \in C_{l} \backslash\left\{\alpha_{l}\right\}$.

Stage 3. Any player $i \in C_{l} \backslash\left\{\alpha_{l}\right\}$ accepts the offer of $\alpha_{l}$ if and only if $y_{j}^{\alpha_{l}} \geq$ $\phi_{j}\left(N \backslash\left\{\alpha_{l}\right\}, C_{-\alpha_{l}}, v_{-\alpha_{l}}\right)$ for every $j \in C_{l} \backslash\left\{\alpha_{l}\right\}$.

If some offer is rejected, for instance, the offer of $\alpha_{l_{0}}$, we go to the subgame where all players other than $\alpha_{l_{0}}$ play this mechanism in $\left(N \backslash\left\{\alpha_{l_{0}}\right\}, C_{-\alpha_{l_{0}}}, v_{-\alpha_{l_{0}}}\right)$. We assume that players in $N \backslash\left\{\alpha_{l_{0}}\right\}$ play according to the strategies profiles of some SPNE with payoff associated $\phi\left(N \backslash\left\{\alpha_{l_{0}}\right\}, C_{-\alpha_{l_{0}}}, v_{-\alpha_{l_{0}}}\right)$ (by induction hypothesis we can find such SPNE).

- Round 2. We assume that players of $N^{r}$ play according to the strategies described in Pérez-Castrillo and Wettstein (2001) when they construct, for any zero-monotonic game, an SPNE that yields the Shapley value of this game as an payoff outcome.

First, we prove that according to these strategies any player $i \in N$ receives as payoff the Owen value $\phi_{i}(N, C, v)$. We must note that the offer of any $\alpha_{l}$ is accepted. Then player $\alpha_{l}$ goes to Round 2 as the representative of $C_{l}$.

Any player $i \in C_{l} \backslash\left\{\alpha_{l}\right\}$ obtains $b_{i}^{\alpha_{l}}+y_{i}^{\alpha_{l}}=$

$$
\phi_{i}(N, C, v)-\phi_{i}\left(N \backslash\left\{\alpha_{l}\right\}, C_{-\alpha_{l}}, v_{-\alpha_{l}}\right)+\phi_{i}\left(N \backslash\left\{\alpha_{l}\right\}, C_{-\alpha_{l}}, v_{-\alpha_{l}}\right)=\phi_{i}(N, C, v) .
$$

We now compute the payoff of any representative $r_{l}$. As $v$ is superadditive we have that $v^{r}$ is zero-monotonic. By Pérez-Castrillo and Wettstein (2001), we know that the 
payoff obtained by $r_{l}$ in Round $2\left(p_{r_{l}}^{2}\right)$ coincides with the Shapley value of $\left(N^{r}, v^{r}\right)$. Then the final payoff obtained by $r_{l}$ is

$$
\begin{aligned}
p_{r_{l}}^{1}+p_{r_{l}}^{2}= & -\sum_{i \in C_{l} \backslash\left\{r_{l}\right\}} b_{i}^{r_{l}}-\sum_{i \in C_{l} \backslash\left\{r_{l}\right\}} y_{i}^{r_{l}}+\phi_{r_{l}}\left(N^{r}, v^{r}\right) \\
= & -\sum_{i \in C_{l} \backslash\left\{r_{l}\right\}}\left(\phi_{i}(N, C, v)-\phi_{i}\left(N \backslash\left\{r_{l}\right\}, C_{-r_{l}}, v_{-r_{l}}\right)\right) \\
& -\sum_{i \in C_{l} \backslash\left\{r_{l}\right\}} \phi_{i}\left(N \backslash\left\{r_{l}\right\}, C_{-r_{l}}, v_{-r_{l}}\right)+\phi_{C_{l}}\left(C, v^{C}\right) \\
= & -\sum_{i \in C_{l} \backslash\left\{r_{l}\right\}} \phi_{i}(N, C, v)+\sum_{i \in C_{l}} \phi_{i}(N, C, v) \\
= & \phi_{r_{l}}(N, C, v) .
\end{aligned}
$$

We now prove that these strategies are an SPNE. As $v$ is superadditive, $\left(N^{r}, v^{r}\right)$ is always zero-monotonic. By Pérez-Castrillo and Wettstein (2001) we conclude that in the subgames obtained after Round 2 these strategies induce an SPNE.

By induction hypothesis, in all the subgames obtained after the rejection of the offer of some proposer $\alpha_{l}$, these strategies induce an SPNE.

We only have to prove that these strategies induce an SPNE in the bidding mechanism associated to any coalition $C_{l}$.

Stage 3. Assume that player $i$ rejects the offer of $\alpha_{l}$. Then the coalitional bidding mechanism of $\left(N \backslash\left\{\alpha_{l}\right\}, C_{-\alpha_{l}}, v_{-\alpha_{l}}\right)$ is played and, by induction hypothesis, after the rejection player $i$ can obtain at most $\phi_{i}\left(N \backslash\left\{\alpha_{l}\right\}, C_{-\alpha_{l}}, v_{-\alpha_{l}}\right)$. Hence, if player $i$ rejects the offer of $\alpha_{l}$, he obtains, at most,

$$
b_{i}^{\alpha_{l}}+\phi_{i}\left(N \backslash\left\{\alpha_{l}\right\}, C_{-\alpha_{l}}, v_{-\alpha_{l}}\right)=\phi_{i}(N, C, v) .
$$

This means that player $i$ does not improve his payoff.

Stage 2. If player $\alpha_{l}$ offers to some player $i \in C_{l}$ less than $\phi_{i}\left(N \backslash\left\{\alpha_{l}\right\}, C_{-\alpha_{l}}, v_{-\alpha_{l}}\right)$, the offer is rejected and, therefore, player $\alpha_{l}$ obtains a final payoff of

$$
v\left(\alpha_{l}\right)-\sum_{i \in C_{l} \backslash\left\{\alpha_{l}\right\}}\left(\phi_{i}(N, C, v)-\phi_{i}\left(N \backslash\left\{\alpha_{l}\right\}, C_{-\alpha_{l}}, v_{-\alpha_{l}}\right)\right) .
$$

By Proposition 1, this payoff is not larger than $\phi_{\alpha_{l}}(N, C, v)$, which means that player $\alpha_{l}$ does not improve his payoff.

If player $\alpha_{l}$ offers to any player $i \in C_{l} \backslash\left\{\alpha_{l}\right\}$ at least $\phi_{i}\left(N \backslash\left\{\alpha_{l}\right\}, C_{-\alpha_{l}}, v_{-\alpha_{l}}\right)$, the offer is accepted. It is straightforward to prove that player $\alpha_{l}$ obtains at most $\phi_{\alpha_{l}}(N, C, v)$. 
Stage 1. First, we prove that for any $i \in C_{l} \in C, B^{i}=0$.

$$
\begin{aligned}
B^{i}= & \sum_{j \in C_{l} \backslash\{i\}} b_{j}^{i}-\sum_{j \in C_{l} \backslash\{i\}} b_{i}^{j} \\
= & \sum_{j \in C_{l} \backslash\{i\}}\left(\phi_{j}(N, C, v)-\phi_{j}\left(N \backslash\{i\}, C_{-i}, v_{-i}\right)\right) \\
& -\sum_{j \in C_{l} \backslash\{i\}}\left(\phi_{i}(N, C, v)-\phi_{i}\left(N \backslash\{j\}, C_{-j}, v_{-j}\right)\right) .
\end{aligned}
$$

As the Owen value satisfies balanced contributions among players, we have that for any $j \in C_{l} \backslash\{i\}$,

$$
\phi_{i}(N, C, v)-\phi_{i}\left(N \backslash\{j\}, C_{-j}, v_{-j}\right)=\phi_{j}(N, C, v)-\phi_{j}\left(N \backslash\{i\}, C_{-i}, v_{-i}\right)
$$

and hence $B^{i}=0$.

Assume that player $i \in C_{l}$ makes a different bid $b^{*}$. If $B^{* i}<0$, the proposer will be another player of $C_{l}$. Then player $i$ can not increase his payoff.

If $B^{\star i}>0$, he becomes the proposer but he must pay $\sum_{j \in C_{l} \backslash\{i\}} b_{j}^{\star i}$ to the other players of $C_{l} \backslash\{i\}$. It is straightforward to prove that player $i$ can obtain, at most, a final payoff of

$$
\phi_{i}(N, C, v)-\sum_{j \in C_{l} \backslash\{i\}} b_{j}^{\star i}+\sum_{j \in C_{l} \backslash\{i\}} b_{j}^{i}
$$

which is smaller than $\phi_{i}(N, C, v)$.

If $B^{\star i}=0$ and player $i$ is not the proposer, using similar arguments to those used when $B^{\star i}<0$, we can conclude that player $i$ does not increase his payoff. If $B^{\star i}=0$ and player $i$ is the proposer, using similar arguments to those used when $B^{\star i}>0$ we can conclude that player $i$ does not increase his payoff.

There exist superadditive games such that the associated coalitional bidding mechanisms have SPNE outcomes whose payoff is different from the Owen value.

Example 1. Consider $(N, C, v)$, where $N=\{1,2,3,4\}, C=\left\{C_{1}, C_{2}\right\}, C_{1}=\{1,2\}$, $C_{2}=\{3,4\}$. Moreover, $v$ is the characteristic function associated to the weighted majority game where the quota is 3 and the weights are 1, 1, 1, and 2 respectively. This means that $v(S)=1$ if and only if $S$ contains some of the following subsets: $\{1,2,3\},\{1,4\}$, $\{2,4\}$, or $\{3,4\}$. 
It is straightforward to prove that

$$
\begin{aligned}
\phi(N, C, v) & =\left(0,0, \frac{1}{2}, \frac{1}{2}\right) \\
\phi\left(N \backslash\{1\}, C_{-1}, v_{-1}\right) & =\left(-, 0, \frac{1}{4}, \frac{3}{4}\right) \\
\phi\left(N \backslash\{2\}, C_{-2}, v_{-2}\right) & =\left(0,-, \frac{1}{4}, \frac{3}{4}\right) \\
\phi\left(N \backslash\{3\}, C_{-3}, v_{-3}\right) & =\left(\frac{1}{4}, \frac{1}{4},-\frac{1}{2}\right) \\
\phi\left(N \backslash\{4\}, C_{-4}, v_{-4}\right) & =\left(\frac{1}{4}, \frac{1}{4}, \frac{1}{2},-\right) .
\end{aligned}
$$

We now define an SPNE whose payoff outcome is $\left(0,0, \frac{1}{4}, \frac{3}{4}\right)$.

Round 1. First, we describe the strategies of players 1 and 2. The bids are $b_{2}^{1}=b_{1}^{2}=0$. Then, the proposer $\alpha_{1}$ is randomly chosen between 1 and 2. Moreover, $y_{j}^{\alpha_{1}}=0$ and player $j$ accepts the offer of $\alpha_{1}$ if and only if $\alpha_{1}$ offers him something positive.

We now describe the strategies of players 3 and 4 . In the subgame obtained after the offer of $\alpha_{1}$ is accepted, the strategies of players 3 and 4 coincide with the strategies whose payoff outcome is the Owen value. We know that these strategies exist by Proposition 2. In the subgame obtained after the offer of $\alpha_{1}$ is rejected, the strategies of players 3 and 4 coincide with the strategies whose payoff outcome is the Owen value of $\left(N \backslash\left\{\alpha_{1}\right\}, C_{-\alpha_{1}}, v_{-\alpha_{1}}\right)$.

Round 2. We assume that players of $N^{r}$ play according to the strategies described in Pérez-Castrillo and Wettstein (2001), which implement the Shapley value.

It is not difficult to check that these strategies are an SPNE.

According to these strategies, the offer of player $\alpha_{1}$ is rejected, which means that player $\alpha_{1}$ obtains a final payoff of $v\left(\alpha_{1}\right)=0$. Then players of $N \backslash\left\{\alpha_{1}\right\}$ obtain as final payoff $\phi\left(N \backslash\left\{\alpha_{1}\right\}, C_{-\alpha_{1}}, v_{-\alpha_{1}}\right)$. This means that the final payoff induced by these strategies is $\left(0,0, \frac{1}{4}, \frac{3}{4}\right)$.

If we want to implement the Owen value, we have to make more assumptions. We make two kind of assumptions: first, about players's behavior in Round 1, and second, about the class of cooperative games.

About players's behavior Moldovanu and Winter (1994) say, "We assume that each player prefers to be a member of large coalitions rather than smaller ones provided that he earns the same payoff in the two agreements". Hart and Mas-Colell (1996) say, "To facilitate exposition we will assume that both proposers and respondents break ties in favor of quick termination of the game". 
If we make in our framework the same assumption that Moldovanu and Winter (1994), we implement the Owen value. The same happens with the assumption by Hart and MasColell (1996).

In order to simplify the exposition, in our model we suppose that if some offer $y^{\alpha}$ of proposer $\alpha$ in Round 1 is rejected by player $j$, then both players ( $\alpha$ and $j$ ) have a "punishment" $\varepsilon>0$ where $\varepsilon$ is very small. Note that with this punishment players prefer large coalitions rather than smaller ones and "both proposers and respondents break ties in favor of quick termination of the game". We call this modification the $\varepsilon$-CBM.

We now define the $\varepsilon$-CBM formally. The structure of the non-cooperative game, bids and offers is the same in CBM and $\varepsilon$-CBM. This means that the strategies available for players are the same in both games. The only difference between CBM and $\varepsilon$-CBM lies on the following aspect of the payoff function in Round 1. Assume that for any $C_{l} \in C, l<l_{0}$ the offer of player $\alpha_{l}$ is accepted and the offer of $\alpha_{l_{0}}$ is rejected by player $j$. Then $\alpha_{l_{0}}$ leaves the non-cooperative game obtaining $v\left(\left\{\alpha_{l_{0}}\right\}\right)-\sum_{i \in C_{l_{0}} \backslash\left\{\alpha_{l_{0}}\right\}} b_{i}^{\alpha_{l_{0}}}-\varepsilon$ (in CBM, player $\alpha_{l_{0}}$ leaves the non-cooperative game obtaining $\left.v\left(\left\{\alpha_{l_{0}}\right\}\right)-\sum_{i \in C_{l_{0}} \backslash\left\{\alpha_{l_{0}}\right\}} b_{i}^{\alpha_{l_{0}}}\right)$. Player $j \in C_{l_{0}} \backslash\left\{\alpha_{l_{0}}\right\}$ obtains as final payoff the sum of $b_{j}^{\alpha_{l_{0}}}-\varepsilon$, and the payoff outcome of the mechanism corresponding to $\left(N^{\prime}, C^{\prime}, v^{\prime}\right)$ (in CBM player $j$ obtains as final payoff the sum of $b_{j}^{\alpha_{l_{0}}}$ and the payoff outcome of the mechanism corresponding to $\left.\left(N^{\prime}, C^{\prime}, v^{\prime}\right)\right)$.

Theorem 1. For any $\varepsilon>0$, the $\varepsilon$-CBM implements the Owen value in SPNE for superadditive games.

Proof. We proceed by induction on the number of players. If there is only one player the result is trivial. Assume that if there is at most $n-1$ players the $\varepsilon$-CBM implements the Owen value in SPNE and, moreover, all the offers of Round 1 are accepted. We now prove that the same holds when there are $n$ players.

Consider the strategies defined as in Proposition 2 but with $b_{j}^{i}=\phi_{j}(N, C, v)-\phi_{j}(N \backslash$ $\left.\{i\}, C_{-i}, v_{-i}\right)+\varepsilon$ and $y_{j}^{\alpha_{l}}=\phi_{j}\left(N \backslash\left\{\alpha_{l}\right\}, C_{-\alpha_{l}}, v_{-\alpha_{l}}\right)-\varepsilon$ for any $C_{l} \in C, i \in C_{l}$, and $j \in C_{l} \backslash\{i\}$. Using similar arguments to those used in the proof of Proposition 2, we conclude that these strategies are an SPNE whose payoff outcome is $\phi(N, C, v)$.

We now prove that the payoff in all SPNE outcomes coincides with the Owen value. We do it in several steps.

The structure of this proof is similar to that of the main result by Pérez-Castrillo and Wettstein (2001). The proof of Step $A$ is completely different. The proof of steps $B, C$, and $D$ is similar although the computations are different. 
Step $A$. At every SPNE outcome, and for every $C_{l} \in C$, the offer of the proposer $\alpha_{l}$ to each player $i \in C_{l} \backslash\left\{\alpha_{l}\right\}$ is $y_{i}^{\alpha_{l}}=\phi_{i}\left(N \backslash\left\{\alpha_{l}\right\}, C_{-\alpha_{l}}, v_{-\alpha_{l}}\right)-\varepsilon$ and every $i \in C_{l} \backslash\left\{\alpha_{l}\right\}$ accepts this offer.

Assume that in each coalition $C_{l} \in\left\{C_{1}, \ldots, C_{m-1}\right\}$, the offer of a proposer $\alpha_{l} \in C_{l}$ is accepted, and consider the subgame starting with the last coalition $C_{m}$. Let $\alpha_{m} \in C_{m}$ be the proposer in $C_{m}$. Let $y^{\alpha_{m}}$ be an offer of $\alpha_{m}$. Let the order of reply of the players in $C_{m} \backslash\left\{\alpha_{m}\right\}$ be $i_{1}, \ldots, i_{k}$.

Claim 1: At every SPNE, the strategies of the players in $C_{m} \backslash\left\{\alpha_{m}\right\}$ must be as follows:

(i) If $y_{i}^{\alpha_{m}}>\phi_{i}\left(N \backslash\left\{\alpha_{m}\right\}, C_{-\alpha_{m}}, v_{-\alpha_{m}}\right)-\varepsilon$ for every $i \in C_{m} \backslash\left\{\alpha_{m}\right\}$, then every $i \epsilon$ $C_{m} \backslash\left\{\alpha_{m}\right\}$ accepts $y^{\alpha_{m}}$.

(ii) If $y_{j}^{\alpha_{m}}<\phi_{j}\left(N \backslash\left\{\alpha_{m}\right\}, C_{-\alpha_{m}}, v_{-\alpha_{m}}\right)-\varepsilon$ for some $j \in C_{m} \backslash\left\{\alpha_{m}\right\}$, then some player in $C_{m} \backslash\left\{\alpha_{m}\right\}$ rejects $y^{\alpha_{m}}$.

(i) Consider the strategy of the last player $i_{k}$. Assuming that his decision node is reached, if he accepts the offer $y^{\alpha_{m}}$, then he receives $b_{i_{k}}^{\alpha_{m}}+y_{i_{k}}^{\alpha_{m}}$, whereas if he rejects $y^{\alpha_{m}}$, then by the induction hypothesis he obtains $b_{i_{k}}^{\alpha_{m}}+\phi_{i_{k}}\left(N \backslash\left\{\alpha_{m}\right\}, C_{-\alpha_{m}}, v_{-\alpha_{m}}\right)-\varepsilon$. Hence, at any SPNE, if $y_{i_{k}}^{\alpha_{m}}>\phi_{i_{k}}\left(N \backslash\left\{\alpha_{m}\right\}, C_{-\alpha_{m}}, v_{-\alpha_{m}}\right)-\varepsilon$, then $i_{k}$ accepts the offer. Repeating the same argument backwards, we can show that players $i_{k-1}, \ldots, i_{1}$ accept the offer.

(ii) Suppose, to the contrary, that there exists $j \in C_{m} \backslash\left\{\alpha_{m}\right\}$ with $y_{j}^{\alpha_{m}}<\phi_{j}(N \backslash$ $\left.\left\{\alpha_{m}\right\}, C_{-\alpha_{m}}, v_{-\alpha_{m}}\right)-\varepsilon$, but all the players in $C_{m} \backslash\left\{\alpha_{m}\right\}$ accept the offer $y^{\alpha_{m}}$. Then, player $j$ receives $b_{j}^{\alpha_{m}}+y_{j}^{\alpha_{m}}$. However, if player $j$ deviates and rejects the offer, then he obtains $b_{j}^{\alpha_{m}}+\phi_{j}\left(N \backslash\left\{\alpha_{m}\right\}, C_{-\alpha_{m}}, v_{-\alpha_{m}}\right)-\varepsilon$, which is greater than $b_{j}^{\alpha_{m}}+y_{j}^{\alpha_{m}}$. Hence, the strategies of the players in $C_{m} \backslash\left\{\alpha_{m}\right\}$ cannot constitute an SPNE.

Claim 2: At every SPNE outcome, every $i \in C_{m} \backslash\left\{\alpha_{m}\right\}$ accepts the offer of the proposer $\alpha_{m}$.

Suppose, to the contrary, that at some SPNE outcome, there exists $i \in C_{m} \backslash\left\{\alpha_{m}\right\}$ who rejects the offer $y^{\alpha_{m}}$. Then, the proposer obtains

$$
v\left(\left\{\alpha_{m}\right\}\right)-\varepsilon-\sum_{i \in C_{m} \backslash\left\{\alpha_{m}\right\}} b_{i}^{\alpha_{m}} .
$$

Let $\delta>0$ be given. For each $i \in C_{m} \backslash\left\{\alpha_{m}\right\}$, define $z_{i}^{\alpha_{m}}(\delta)$ by

$$
z_{i}^{\alpha_{m}}(\delta)=\phi_{i}\left(N \backslash\left\{\alpha_{m}\right\}, C_{-\alpha_{m}}, v_{-\alpha_{m}}\right)-\varepsilon+\delta
$$

Suppose that the proposer $\alpha_{m}$ proposes $z^{\alpha_{m}}(\delta)$. By Claim 1 (i), for any $\delta>0$, every $i \in C_{m} \backslash\left\{\alpha_{m}\right\}$ accepts $z^{\alpha_{m}}(\delta)$. Hence, player $\alpha_{m}$ is the representative of coalition $C_{m}$ in Round 2. Now, in Round 2, there are $m$ players $\left\{\alpha_{1}, \ldots, \alpha_{m}\right\}$, where, for any coalition 
$C_{l} \in C, \alpha_{l}$ is the representative of coalition $C_{l}$. As the representatives are playing an SPNE of the bidding mechanism associated to $\left(N^{r}, v^{r}\right)$, by Pérez-Castrillo and Wettstein $(2001)$ we know that the payoff obtained by player $\alpha_{m}$ in Round 2 is $\phi_{\alpha_{m}}\left(N^{r}, v^{r}\right)=\phi_{C_{m}}\left(C, v^{C}\right)=$ $\sum_{i \in C_{m}} \phi_{i}(N, C, v)$. Then, the final payoff of player $\alpha_{m}$ is

$$
\begin{aligned}
\sum_{i \in C_{m}} \phi_{i}(N, C, v)-\sum_{i \in C_{m} \backslash\left\{\alpha_{m}\right\}}\left[\phi_{i}\left(N \backslash\left\{\alpha_{m}\right\}, C_{-\alpha_{m}}, v_{-\alpha_{m}}\right)-\varepsilon+\delta\right]-\sum_{i \in C_{m} \backslash\left\{\alpha_{m}\right\}} b_{i}^{\alpha_{m}} \\
=\sum_{i \in C_{m}} \phi_{i}(N, C, v)-\sum_{i \in C_{m} \backslash\left\{\alpha_{m}\right\}} \phi_{i}\left(N \backslash\left\{\alpha_{m}\right\}, C_{-\alpha_{m}}, v_{-\alpha_{m}}\right) \\
+\left(\left|C_{m}\right|-1\right) \varepsilon-\left(\left|C_{m}\right|-1\right) \delta-\sum_{i \in C_{m} \backslash\left\{\alpha_{m}\right\}} b_{i}^{\alpha_{m}} .
\end{aligned}
$$

By Proposition 1, we know that

$$
a=\sum_{i \in C_{m}} \phi_{i}(N, C, v)-\sum_{i \in C_{m} \backslash\left\{\alpha_{m}\right\}} \phi_{i}\left(N \backslash\left\{\alpha_{m}\right\}, C_{-\alpha_{m}}, v_{-\alpha_{m}}\right)-v\left(\left\{\alpha_{m}\right\}\right) \geq 0 .
$$

Then, if $0<\delta<\frac{a+\left|C_{m}\right| \varepsilon}{\left(\left|C_{m}\right|-1\right)}$, the payoff of $\alpha_{m}$ obtained by offering $z^{\alpha_{m}}(\delta)$ is greater than that obtained by offering $y^{\alpha_{m}}$. Hence, to offer $y^{\alpha_{m}}$ cannot be an SPNE strategy of the proposer $\alpha_{m}$, which is a contradiction.

Claim 3: At every SPNE, and for every $i \in C_{m} \backslash\left\{\alpha_{m}\right\}$,

$$
y_{i}^{\alpha_{m}}=\phi_{i}\left(N \backslash\left\{\alpha_{m}\right\}, C_{-\alpha_{m}}, v_{-\alpha_{m}}\right)-\varepsilon
$$

Let $y^{\alpha_{m}}$ be the offer of $\alpha_{m}$ at an SPNE. By Claim 2, $y^{\alpha_{m}}$ must be accepted by every $i \in C_{m} \backslash\left\{\alpha_{m}\right\}$. Then, it follows from Claim 1 (ii) that for every $i \in C_{m} \backslash\left\{\alpha_{m}\right\}$, $y_{i}^{\alpha_{m}} \geq \phi_{i}\left(N \backslash\left\{\alpha_{m}\right\}, C_{-\alpha_{m}}, v_{-\alpha_{m}}\right)-\varepsilon$. Suppose that for some $j \in C_{m} \backslash\left\{\alpha_{m}\right\}, y_{j}^{\alpha_{m}}>$ $\phi_{j}\left(N \backslash\left\{\alpha_{m}\right\}, C_{-\alpha_{m}}, v_{-\alpha_{m}}\right)-\varepsilon$. Let $\tau=y_{j}^{\alpha_{m}}-\left[\phi_{j}\left(N \backslash\left\{\alpha_{m}\right\}, C_{-\alpha_{m}}, v_{-\alpha_{m}}\right)-\varepsilon\right]>0$. For each $i \in C_{m} \backslash\left\{\alpha_{m}\right\}$, define $w_{i}^{\alpha_{m}}=\phi_{i}\left(N \backslash\left\{\alpha_{m}\right\}, C_{-\alpha_{m}}, v_{-\alpha m}\right)-\varepsilon+\frac{\tau}{\left|C_{m}\right|}$. Suppose that the proposer $\alpha_{m}$ deviates and offers $w^{\alpha_{m}}$. Then, by Claim 1 (i), every $i \in C_{m} \backslash\left\{\alpha_{m}\right\}$ accepts $w^{\alpha_{m}}$. Moreover, since

$$
\begin{aligned}
\sum_{i \in C_{m} \backslash\left\{\alpha_{m}\right\}} w_{i}^{\alpha_{m}} & =\sum_{i \in C_{m} \backslash\left\{\alpha_{m}\right\}}\left[\phi_{i}\left(N \backslash\left\{\alpha_{m}\right\}, C_{-\alpha_{m}}, v_{-\alpha_{m}}\right)-\varepsilon\right]+\frac{\left|C_{m}\right|-1}{\left|C_{m}\right|} \tau \\
& <\sum_{i \in C_{m} \backslash\left\{\alpha_{m}\right\}} y_{i}^{\alpha_{m}},
\end{aligned}
$$

the proposer $\alpha_{m}$ obtains a greater payoff by offering $w^{\alpha_{m}}$ than by offering $y^{\alpha_{m}}$. Hence, to offer $y^{\alpha_{m}}$ cannot be an SPNE strategy, which is a contradiction.

Repeating the same arguments for coalitions $C_{m-1}, \ldots, C_{1}$, we can prove Step $A$. 
Step $B$. Assume that we are in Stage 1 of Round 1 of the bidding mechanism associated to $C_{l} \in C$. Then in any SPNE, $B^{i}=0$ for any $i \in C_{l}$.

It is straightforward to prove that $\sum_{i \in C_{l}} B^{i}=0$. We take $X=\left\{i \in C_{l} \mid B^{i}=\max _{j \in C_{l}} B^{j}\right\}$. If $X=C_{l}$, the result holds because $\sum_{i \in C_{l}} B^{i}=0$.

If $X \neq C_{l}$, we get a contradiction by proving that player $i \in X$ has a deviation which improves his final payoff. We take $j \in C_{l} \backslash X$ such that $B^{j} \geq B^{k}$ for any $k \in C_{l} \backslash X$. Assume that player $i$ makes a new bid $b^{\prime i}$, where $b_{k}^{i i}=b_{k}^{i}+\delta$ if $k \in X \backslash\{i\}, b_{k}^{\prime j}=b_{k}^{j}-|X| \delta$, and $b_{k}^{i i}=b_{k}^{i}$ if $k \in C_{l} \backslash(X \cup\{j\})$.

For any $k \in C_{l}$, we compute $B^{\prime k}$ assuming that $b^{\prime k}=b^{k}$ for any $k \in C_{l} \backslash\{i\}$. Then $B^{\prime k}=B^{k}-\delta$ if $k \in X, B^{\prime j}=B^{j}+|X| \delta$, and $B^{\prime k}=B^{k}$ if $k \in C_{l} \backslash(X \cup\{j\})$.

Since $B^{j}<B^{i}$, we can find $\delta>0$ satisfying $B^{j}+|X| \delta<B^{i}-\delta$. Moreover, $X^{\prime}=$ $\left\{k \in C_{l} \mid B^{\prime k}=\max _{h \in C_{l}} B^{\prime h}\right\}=X$. This means that any player of $X$ is the proposer with the same probability under $b^{i}$ and $b^{i}$. When player $i$ is not the proposer, which happens with probability $\frac{|X|-1}{|X|}$, he obtains, by Step $A$, the same making a bid $b^{i}$ or $b^{i}$. But if player $i$ is the proposer, which happens with probability $\frac{1}{|X|}$, he obtains, by Step $A, \delta$ units more with $b^{i}$ than with $b^{i}$.

Step $C$. Assume that we are in Stage 1 of Round 1 of the bidding mechanism associated to $C_{l} \in C$. Then, at every SPNE, the payoff of any player $i \in C_{l}$ is the same regardless of who is chosen as the proposer.

By Step $B$, we know that $B^{i}=0$ for any $i \in C_{l}$.

Assume that some player $i$ strictly prefers to be (not to be) the proposer. Then player $i$ can improve his payoff by slightly increasing (decreasing) one of his bids $b_{j}^{i}$. But this is impossible in an SPNE.

Step $D$. In any SPNE outcome of $\epsilon$-CBM any player $i \epsilon N$ obtains as final payoff his Owen value.

Assume that players are playing according to some SPNE. Given $i \in C_{l} \in C$, we denote by $p_{i}$ the final payoff obtained by player $i$ in this SPNE.

By Step $B$, we know that any player of $C_{l}$ is the proposer with probability $\frac{1}{\left|C_{l}\right|}$.

If player $i$ is the proposer, we know, by Step $A$, that his final payoff is

$$
\sum_{j \in C_{l}} \phi_{j}(N, C, v)-\sum_{j \in C_{l} \backslash\{i\}} \phi_{j}\left(N \backslash\{i\}, C_{-i}, v_{-i}\right)+\left(\left|C_{l}\right|-1\right) \epsilon-\sum_{j \in C_{l} \backslash\{i\}} b_{j}^{i} .
$$

If $j \in C_{l} \backslash\{i\}$ is the proposer then the final payoff of player $i$ is, by Step $A$,

$$
b_{i}^{j}+\phi_{i}\left(N \backslash\{j\}, C_{-j}, v_{-j}\right)-\epsilon .
$$


By Step $C$, we know that $\left|C_{l}\right| p_{i}=$

$$
\begin{aligned}
\sum_{j \in C_{l}} \phi_{j}(N, C, v) & -\sum_{j \in C_{l} \backslash\{i\}} \phi_{j}\left(N \backslash\{i\}, C_{-i}, v_{-i}\right)+\left(\left|C_{l}\right|-1\right) \epsilon-\sum_{j \in C_{l} \backslash\{i\}} b_{j}^{i} \\
& +\sum_{j \in C_{l} \backslash\{i\}}\left(b_{i}^{j}+\phi_{i}\left(N \backslash\{j\}, C_{-j}, v_{-j}\right)-\epsilon\right) .
\end{aligned}
$$

By Step $B$, we know that

$$
-\sum_{j \in C_{l} \backslash\{i\}} b_{j}^{i}+\sum_{j \in C_{l} \backslash\{i\}} b_{i}^{j}=-B^{i}=0 .
$$

Hence, $\left|C_{l}\right| p_{i}=$

$$
\sum_{j \in C_{l} \backslash\{i\}}\left(\phi_{i}\left(N \backslash\{j\}, C_{-j}, v_{-j}\right)-\phi_{j}\left(N \backslash\{i\}, C_{-i}, v_{-i}\right)\right)+\sum_{j \in C_{l}} \phi_{j}(N, C, v) .
$$

Since the Owen value satisfies the property of balanced contributions among coalitions, we have that

$$
\begin{aligned}
\left|C_{l}\right| p_{i} & =\sum_{j \in C_{l} \backslash\{i\}}\left(\phi_{i}(N, C, v)-\phi_{j}(N, C, v)\right)+\sum_{j \in C_{l}} \phi_{j}(N, C, v) \\
& =\left(\left|C_{l}\right|-1\right) \phi_{i}(N, C, v)-\sum_{j \in C_{l} \backslash\{i\}} \phi_{j}(N, C, v)+\sum_{j \in C_{l}} \phi_{j}(N, C, v) \\
& =\left|C_{l}\right| \phi_{i}(N, C, v) .
\end{aligned}
$$

Then $p_{i}=\phi_{i}(N, C, v)$. This finishes the proof of Theorem 1 .

Remark 5. In the $\varepsilon$-CBM, if an offer is rejected, the proposer and the responder who rejects the offer have a punishment. It is easy to check that the result is also true if only proposers (or responders) have a punishment.

Theorem 1 also holds if the punishment to the proposer is agent-dependent, $i$. e. any agent $i$ has a punishment $\varepsilon_{i}>0$ for being removed from the game.

We have just proved that if we make assumptions about player's behavior, which appears in the $\varepsilon$-CBM, we can implement the Owen value in the class of superadditive games.

As we said before, another way to avoid the multiplicity of payoffs associated to SPNE outcomes in the CBM associated to superadditive games is to find a subclass of such games where the Owen value is the unique payoff associated to SPNE outcomes. We have the following result:

Theorem 2. The CBM implements the Owen value in SPNE for strictly superadditive games. 
Proof. We already know, by Proposition 2, that there is an SPNE outcome of CBM whose payoff is the Owen value.

Using similar arguments to those used in the proof of Theorem 1 we can prove that the payoff associated to every SPNE outcome coincides with the Owen value.

Remark 6. A natural question that arises is: what happens if in Round 1 coalitions announce nothing? (in CBM they announce if there is an agreement or some player is removed). This means that players in a coalition have no information about what happens in other coalitions. Later, in Round 2, the representative of any coalition announces to the other representatives the resources that he has. We call this non-cooperative game the "simultaneous coalitional bidding mechanism" (SCBM). We also define the $\epsilon$-SCBM in the same way that we have done with the $\epsilon$-CBM.

Note that the only subgames in SCBM and $\epsilon$-SCBM are the whole game and those obtained after Round 2.

Using similar arguments to those used in Proposition 2, it is easy to prove that the Owen value is the payoff associated to some SPNE outcome of SCBM and $\epsilon$-SCBM.

Nevertheless, we have no uniqueness as we can see in the following example, which works in SCBM and $\epsilon$-SCBM. We take $(N, C, v)$, where $N=\{1,2,3,4\}, C=\left\{C_{1}, C_{2}\right\}$, $C_{1}=\{1,2\}, C_{2}=\{3,4\}, v(S)=100$ if $|S|=4$, and $v(S)=0$ if $|S|<4$.

We consider the following strategies:

Round 1. Players of $C_{1}$ play as follows: in Stage $1 b_{2}^{1}=b_{1}^{2}=0$; in Stage 2 the proposer, $\alpha_{1}$, offers $y_{i}^{\alpha_{1}}=0$ if $i \neq 1$ and $y_{i}^{\alpha_{1}}=50$ if $i=1$, any player $j \in C_{1} \backslash\left\{\alpha_{1}\right\}$ accepts an offer $y$ if and only if $y_{i}^{\alpha_{1}}=0$ if $i \neq 1$ and $y_{i}^{\alpha_{1}}=50$ if $i=1$. Players of $C_{2}$ play according to the strategies defined in Proposition 2.

Round 2. The representatives play according to the strategies described in PérezCastrillo and Wettstein (2001), which implement the Shapley value.

It is not difficult to prove that these strategies are an SPNE. Moreover, they induce as final payoff $(50,0,25,25)$. The Owen value is $(25,25,25,25)$.

\section{Concluding remarks}

In this paper we define the coalitional bidding mechanism, which generalizes the bidding mechanism of Pérez-Castrillo and Wettstein (2001) for situations where players are partitioned into fixed groups. We prove that for superadditive games there always exists an SPNE whose payoff outcome coincides with the Owen value. However, unlike the result of 
Pérez-Castrillo and Wettstein (2001) on implementation of the Shapley value, there exist SPNE whose payoff outcome is different from the Shapley value. But if we restrict the behavior of agents (as in Moldovanu and Winter (1994) or Hart and Mas-Colell (1996)) or we restrict the class of games (to strictly superadditive games), we can implement the Owen value.

\section{References}

Calvo E., Lasaga J., Winter E. (1996) The principle of balanced contributions and hierarchies of cooperation. Mathematical Social Sciences 31: 171-182.

Carreras F., Owen G. (1988) Evaluation of the Catalonian parliament 1980-1984. Mathematical Social Sciences 15: 87-92.

Gul F. (1989) Bargaining foundations of Shapley value. Econometrica 57: 81-95.

Hart S., Mas-Colell A. (1989) Potential, value, and consistency. Econometrica 57: 589614.

Hart S., Mas-Colell A. (1996) Bargaining and value. Econometrica 64: 357-380.

Moldovanu B., Winter E. (1994) Core implementation and increasing returns to scale for cooperation. Journal of Mathematical Economics 23: 533-548.

Myerson R.B. (1980) Conference structures and fair allocation rules. International Journal of Game Theory 9: 169-182.

Owen G. (1977) Values of games with a priori unions. In: Henn R., Moeschlin O. (eds) Essays in Mathematical Economics and Game Theory, Springer-Verlag, Berlin Heidelberg New York: 76-88.

Pérez-Castrillo D., Wettstein D. (2001) Bidding for the surplus: A non-cooperative approach to the Shapley value. Journal of Economic Theory 100: 274-294.

Shapley S. (1953) A value for n-person games. In: Kuhn H.W., Tucker A.W. (eds) Contributions to the Theory of Games II, Princeton University Press, Princeton NJ: 307-317.

Vázquez-Brage M., Van den Nouweland A., García-Jurado I. (1997) Owen's coalitional value and aircraft landing fees. Mathematical Social Sciences 34: 273-286.

Winter E. (1992) The consistency and potential for values of games with coalition structure. Games and Economic Behavior 4: 132-144. 